\title{
Consenso de tratamiento del síndrome nefrótico en la infancia
}

\author{
Consensus on treatment of nephrotic syndrome in childhood
}

Comité de Nefrología, Sociedad Argentina de Pediatría*

http:/ /dx.doi.org/10.5546/aap.2014.277

\section{INTRODUCCIÓN}

Entre los años 2009 y 2011, el Comité de Nefrología de la Sociedad Argentina de Pediatría llevó a cabo cuatro reuniones ampliadas, en las cuales se consensuaron las definiciones y el tratamiento del síndrome nefrótico primario (SNP).

El SNP es aquel que se desarrolla sin tener una enfermedad causal conocida.

Es el más frecuente en la infancia.

En Argentina, la incidencia es de 1,7 a 1,9 casos nuevos por cada 100000 niños menores de 14 años. ${ }^{1}$

En Estados Unidos, es levemente mayor: 2-7 casos nuevos por 100000 niños menores de 16 años. ${ }^{2}$

La importancia de realizar este consenso de tratamiento para niños con SNP radica en que, a través de todos los años transcurridos (más de 40) desde que se comenzó a tratar esta enfermedad con corticoides, han surgido nuevas drogas inmunosupresoras y distintos esquemas de tratamiento, especialmente para aquellos pacientes que no responden al tratamiento con corticoides o presentan graves efectos adversos a estos.

La primera publicación sobre la eficacia de los corticoides en estos niños data del año 1952. Gavin CA y Wilson $\mathrm{EH}$, trataron a seis pacientes con altas dosis de cortisona (de 100 a $300 \mathrm{mg}$ /día) por 5 días y el resultado fue la remisión de la proteinuria. ${ }^{3}$

En 1978, se publica uno de los primeros estudios multicéntricos prospectivos en nefrología pediátrica con la incorporación de 24 centros de América del Norte, Europa y Asia.

El trabajo duró 7 años (1967-1974) y fue elaborado por la International Study of Kidney Disease in Children (ISKDC). ${ }^{2}$ El estudio incorporó 521 niños con síndrome nefrótico (SN), a quienes se les realizó biopsia luego de un tratamiento con prednisona en dosis de $60 \mathrm{mg} / \mathrm{m}^{2} /$ día (mx. $80 \mathrm{mg} / 24$ h) por 4 semanas, seguido de $40 \mathrm{mg}$ / $\mathrm{m}^{2} /$ día en días alternos por otras 4 semanas.

En 1981, Greifer y Edelman mostraron en su estudio sobre diagnóstico y evolución de niños con síndrome nefrótico el resultado que cambió la conducta con respecto a esta patología: el 92\% de los pacientes (389 niños de entre 1 y 16 años de edad) que respondieron al tratamiento con corticoides presentaban en la biopsia "lesiones mínimas" (microscopía óptica sin alteraciones, inmunofluorescencia negativa, microscopía óptica con fusión pedicelar). ${ }^{4}$

Por esto, actualmente, ante el comienzo de los síntomas, se indica el tratamiento y se realiza la biopsia
Correspondencia: mlaso@intramed.net

Conflicto de intereses: Ninguno que declarar.

Recibido: 4-11-2013 Aceptado: 28-11-2013
* Coordinación: Dra. María del Carmen Laso, Dra. Marta Adragna

Elaboración: Dres. Marta Adragna, Laura Alconcher, Juan Ignacio Ayub, Lidia Ghezzi, María del Carmen Laso, Susana Miceli, Alicia Sierro, Javier Zalba. Participación: Comité Ampliado de Nefrología Pediátrica de la Sociedad Argentina de Pediatría. 
renal en caso de corticorresistencia.

El SNP es una enfermedad que, en la mayoría de los casos, evoluciona con recaídas hasta la pubertad, a veces con corticodependencia, por lo que el uso de corticoides en dosis elevadas y por tiempos prolongados provoca en estos niños graves efectos adversos, que comprometen al nefrólogo pediatra a evaluar otras terapias alternativas con tratamientos libres de ellos, pero que no siempre son efectivas para lograr la remisión de la crisis nefrótica.

Por lo tanto, este consenso surge de la necesidad de aclarar conceptos sobre diagnóstico clínico y tratamiento inmunosupresor entre nefrólogos y pediatras para obtener mejores resultados en beneficio de estos pacientes.

\section{OBJETIVOS}

Adoptar una decisión de común acuerdo entre los miembros del Comité de Nefrología de la Sociedad Argentina de Pediatría para definir síndrome nefrótico, remisión, recaída, recaídas frecuentes, corticodependencia y corticorresistencia.

Trasmitir a todos los profesionales encargados del cuidado de niños que sufren síndrome nefrótico primario una guía consensuada de tratamiento específico.

\section{METODOLOGÍA}

Los miembros del Comité Ejecutivo realizaron una búsqueda sistemática de trabajos internacionales y de las principales guías y consensos elaborados con posterioridad al año 2008.

Los resultados analizados se dieron a conocer en dos reuniones abiertas con los integrantes del Comité Ampliado de Nefrología. En estas reuniones, la concurrencia no fue menor de 50 especialistas y se encontraban presentes los jefes de servicios de todos los hospitales que cuentan con la especialidad, además de algunos representantes del interior del país.

La búsqueda de la bibliografía se realizó a través de Medline y Lilacs.

Los artículos seleccionados para el análisis fueron:

a. Reviews de Cochrane, base de datos obtenida por revisión sistemática de artículos elegidos de manera rigurosa para evaluar la efectividad de tratamiento, 2010. Registro Cochrane de Ensayos Controlados (Cochrane Register of Controlled Trials). 5,6

b. Guías de la Academia Estadounidense de Pediatría 2009.? c. Protocolos actualizados al año 2008. Asociación Española de Pediatría. ${ }^{8}$

d Kidney Disease: improvement global outcome (KDIGO). ${ }^{9}$

e. Datos retrospectivos de centros nefrológicos presentes en el consenso. (Experiencia de tratamiento con ciclofosfamida en pacientes corticorresistentes).

\section{DEFINICIONES}

- Síndrome nefrótico

Pérdida de proteínas en orina mayor o igual ( $\geq$ ) a $50 \mathrm{mg} / \mathrm{kg} / \mathrm{d}$ o $40 \mathrm{mg} / \mathrm{m}^{2} /$ hora o índice proteinuria/creatininuria $(\mathrm{PrU} / \mathrm{CrU})$ en muestra aislada $\geq$ a 2 (expresado en $\mathrm{mg} / \mathrm{mg}$ ) con hipoalbuminemia, definida como albúmina $\leq$ a $2,5 \mathrm{~g} / \mathrm{dl}$, generalmente asociada a edema e hipercolesterolemia.

El edema es el signo clínico más frecuente que sugiere el diagnóstico en el primer episodio, aunque no siempre está presente en las recaídas.

- El edema intersticial es un hallazgo siempre presente en el debut y es el signo clínico más llamativo de las manifestaciones del SN.

- Es blando y deja "godet" o signo de la fóvea. De ubicación variable en el transcurso del día, localizado en las zonas declives.

- Con frecuencia puede llegar a ser masivo (>30\% del peso corporal) y restringir la actividad física.

- Puede comprometer las serosas (ascitis, derrame pleural) y genitales (escroto, vulva).

\section{Fisiopatología del edema}

El factor desencadenante es la retención primaria y/o secundaria de sodio.

La fisiopatología se explica por 2 teorías: 1) la disminución del volumen plasmático (underfill) o 2) su expansión (overfill).

En la primera (underfill), la proteinuria masiva provoca hipoalbuminemia, contracción del volumen arterial efectivo, estimulación mineralocorticoide, retención de sodio a nivel del túbulo distal, expansión del líquido extracelular (LEC) y edema. La hipoalbuminemia causa una disminución de la presión oncótica plasmática; esta disminución ocasionaría un "desequilibrio" en las fuerzas de Starling, produciendo la translocación de fluido del espacio intravascular hacia el espacio intersticial, lo que causa una disminución en el volumen arterial efectivo. Secundario a esto, se activa el sistema renina-angiotensina-aldosterona y el sistema 
nervioso simpático; se incrementa la liberación de hormona antidiurética y se inhibiría la liberación del péptido natriurético auricular. Todo esto produciría la retención de sodio y agua por parte del riñón con la consiguiente aparición del edema.

Sin embargo, diversas observaciones experimentales y clínicas realizadas durante el transcurso de los años no apoyarían esta hipótesis, por lo que se postuló una segunda teoría. El overfill: el aumento de la reabsorción de sodio a nivel del túbulo contorneado distal se produce por la activación de los canales epiteliales de sodio (EcNA), expansión del LEC y edema.

Las últimas investigaciones indicarían que la activación de los EcNA es provocada por la plasmina, presente en la orina de los pacientes que sufren una recaída de $\mathrm{SN} .^{10,11}$

\section{- Etiología del SN}

La ultrafiltración glomerular transcurre a través de la superficie de filtración glomerular compuesta por el endotelio fenestrado, la membrana basal glomerular y las células epiteliales viscerales o podocitos.

La causa de la pérdida masiva de proteínas a través de esta superficie es desconocida.

La hipótesis más aceptada y estudiada es la pérdida de la selectividad de la filtración para la carga y la forma de las sustancias que atraviesan la superficie de filtración glomerular.

La responsable de esta acción sería una linfoquina, sintetizada por los linfocitos $\mathrm{T}$ e investigada desde la década del 70 hasta nuestros días. ${ }^{12,13}$

Durante los dos primeros años de la vida, la alteración estructural de la superficie de filtración provocada por mutaciones genéticas de las proteínas que componen la ultraestructura de la superficie de filtración dan origen a las $2 / 3$ partes del síndrome nefrótico. ${ }^{14}$

Conclusión: la etiología del SNP es desconocida; sin embargo, la teoría inmunológica explicaría la mayoría de los casos de SNP, excepto en los dos primeros años de la vida, en los cuales debe considerarse la causa genética.

\section{- Remisión}

Descenso de la proteinuria a valores fisiológicos $\left(<\right.$ a $5 \mathrm{mg} / \mathrm{kg} / \mathrm{d}$ o $<4 \mathrm{mg} / \mathrm{m}^{2} / \mathrm{h}$ o un índice $\mathrm{PrU} / \mathrm{CrU}<0,20)$ o una tirilla reactiva en orina negativa, durante 3 días consecutivos.

El 80\% de los pacientes presenta remisión luego de un tratamiento inicial con corticoides.

\section{- Recaída}

Reaparición de la proteinuria en rango nefrótico o tirilla reactiva en orina con 2 o más cruces durante 5 días consecutivos o reaparición de edema, sin proceso infeccioso concomitante.

Más del $80 \%$ de los pacientes presentan recaídas en la evolución de la enfermedad. ${ }^{15}$

\section{- Recaídas frecuentes}

Más de 2 recaídas en 6 meses o más de 3 en 1 año.

\section{- Corticodependencia}

Dos recaídas consecutivas al descender los corticoides o dentro de las 2 semanas siguientes a la suspensión de estos.

Ambos grupos de pacientes responden al tratamiento con corticoides y en la práctica clínica no se diferencian en las alternativas de tratamiento propuestas por el consenso.

- Corticorresistencia: es la falta de remisión de la proteinuria luego de un tratamiento con:

- Opción a) prednisona $2 \mathrm{mg} / \mathrm{kg} / \mathrm{d}$ o $60 \mathrm{mg} /$ $\mathrm{m}^{2} / \mathrm{d}$ (dosis máxima, $60 \mathrm{mg}$ ) en días continuos durante 4-6 semanas en 1 dosis diaria a la mañana entre las 8 y las $10 \mathrm{~h}$, seguido de prednisona $1,5 \mathrm{mg} / \mathrm{kg}$ o $40 \mathrm{mg} / \mathrm{m}^{2}$ en días alternos durante 4-6 semanas.

- Opción b) prednisona $60 \mathrm{mg} / \mathrm{m}^{2} / \mathrm{d}$ o $2 \mathrm{mg} /$ $\mathrm{kg} / \mathrm{d}$ durante 4-6 semanas en días continuos más 3 pulsos de metilprednisolona de $10 \mathrm{mg} / \mathrm{kg} /$ dosis.

\section{TRATAMIENTO \\ Objetivos}

Lograr la inducción de la remisión para obtener una mejoría sintomática, es decir: proteinuria en rango fisiológico, recuperación de la diuresis, desaparición de los edemas y normalización de la albuminemia y, en segundo lugar, minimizar los efectos adversos secundarios a los inmunosupresores.

\section{Corticoides}

La principal acción de los glucocorticoides es el efecto antiinflamatorio e inmunosupresor sobre linfocitos T. Ejercen una acción directa sobre las células glomerulares e inmunes: podocitos, células endoteliales, linfocitos T, macrófagos y células dendríticas; inhiben la apoptosis y favorecen su estabilización funcional. Este mecanismo se lleva a cabo vía receptores de los corticoides con inhibición de la activación del factor de transcripción nuclear NF-қ B. ${ }^{16,17}$ 


\section{- Tratamiento inicial}

Prednisona $2 \mathrm{mg} / \mathrm{kg}$ o $60 \mathrm{mg} / \mathrm{m}^{2}$ en días continuos (dosis máxima, $60 \mathrm{mg} / \mathrm{d}$ ) durante $4-6$ semanas, en 1 toma, dosis diaria a la mañana entre las 8 y las $10 \mathrm{~h}$, seguido de prednisona $1,5 \mathrm{mg} / \mathrm{kg}$ o $40 \mathrm{mg} / \mathrm{m}^{2}$ en días alternos durante 4-6 semanas.

Equivalencia: prednisona $1 \mathrm{mg}=$ meprednisona $0,8 \mathrm{mg}$.

En nuestro país es más accesible la meprednisona.

La mayoría de los pacientes corticosensibles remiten la proteinuria entre los 5 y los 7 días del comienzo del tratamiento; son muy pocos los que lo hacen en forma tardía.

La respuesta es clínicamente visible por el aumento significativo de la diuresis, la desaparición de los edemas y la normalización de los parámetros de laboratorio.

La ventaja del tratamiento más largo, 6 semanas de continuo y 6 semanas en días alternos, es la disminución del número de recaídas dentro de los 12 y los 24 meses del debut de la enfermedad, sin aumentar los efectos adversos relacionados con una mayor dosis acumulativa de prednisona. ${ }^{18}$

La sensibilidad inicial a la prednisona se correlaciona en forma directa, en la mayoría de los pacientes, con el patrón histológico de lesiones mínimas.

\section{- Tratamiento de la recaída}

Prednisona $60 \mathrm{mg} / \mathrm{m}^{2}$ o $2 \mathrm{mg} / \mathrm{kg}$ en días continuos (dosis máxima, $60 \mathrm{mg} / \mathrm{m}^{2} / \mathrm{d}$ ) hasta 5 días posteriores a la remisión, seguido de $40 \mathrm{mg}$ / $\mathrm{m}^{2} \mathrm{o} 1,5 \mathrm{mg} / \mathrm{kg}$ en días alternos por 4-6 semanas.

Entre el 80 y el $90 \%$ de los pacientes con SNP recaen una o más veces, y alrededor del $35 \%$ al $50 \%$ presentan recaídas frecuentes o son corticodependientes. ${ }^{19-22}$

El comportamiento de la enfermedad durante los 6 primeros meses luego de haber completado el tratamiento inicial predice el pronóstico a largo plazo. Datos provenientes del ISKDC demostraron que de 148 niños que permanecieron en remisión durante los 6 meses luego del episodio inicial, el $76 \%$ nunca recayó o lo hicieron en forma infrecuente. ${ }^{20}$

\section{- Tratamiento de las recaídas frecuentes y corticodependencia}

Casi no existen trabajos aleatorizados y controlados con regímenes de corticoides en estos pacientes.

El riesgo de recaídas dentro de los dos primeros años desciende un $40 \%$ si el tratamiento inicial con corticoides se realiza durante 3 meses comparado con 2 meses. ${ }^{18}$

De acuerdo con las definiciones, existe una diferencia entre los dos comportamientos, pero los esquemas de tratamiento y sus opciones son las mismas para ambos pacientes.

En definitiva, ambos son corticosensibles y dependen del corticoide para remitir la proteinuria.

Para los pacientes corticodependientes o que presentan recaídas frecuentes -siempre que no existan efectos adversos-, la mejor opción para mantener la remisión son los corticoides.

Por lo tanto, las opciones para la primera indicación son:

a) Prednisona $60 \mathrm{mg} / \mathrm{m}^{2} /$ o $2 \mathrm{mg} / \mathrm{kg} /$ en días continuos hasta 5 días posteriores a la remisión, seguido de $40 \mathrm{mg} / \mathrm{m}^{2} \mathrm{o} 1,5 \mathrm{mg}$ / $\mathrm{kg}$ en días alternos por 4 semanas. A partir de aquí, disminuir el corticoide $25 \%$ de la dosis cada 15 días hasta alcanzar una dosis menor de $0,5 \mathrm{mg} / \mathrm{kg}$ en días alternos, manteniendo el tratamiento durante 6-12 meses. Si el paciente recae durante el descenso de la prednisona:

a.1. Reiniciar el esquema, pero en el descenso final dejar una dosis ligeramente superior a la cual recayó.

a.2. Si la dosis en días alternos en la que se produce la recaída es $\geq$ a $0,5 \mathrm{mg} / \mathrm{kg}$, pasar a $0,25 \mathrm{mg} / \mathrm{kg} /$ día todos los días.

b) Prednisona igual a la opción a), pero incorporar levamisol en la remisión en una dosis de $2,5 \mathrm{mg} / \mathrm{kg}$ en días alternos durante un período de 12-24 meses.

El objetivo es suspender los corticoides antes de los 6 meses.

Antes del tratamiento y durante este, realizar hemograma, hepatograma y función renal.

c) Prednisona igual a la opción a), pero incorporar ciclofosfamida en el momento de la remisión en una dosis de $2 \mathrm{mg} / \mathrm{kg}$ / día (dosis máxima diaria, $100 \mathrm{mg}$ ) durante 8-12 semanas (dosis máxima acumulativa, $180 \mathrm{mg} / \mathrm{kg}$ ).

- Suspender la prednisona lentamente al iniciar la ciclosfamida.

El levamisol es una droga antihelmíntica con propiedades inmunorreguladoras. El mecanismo exacto por el cual ejerce su efecto beneficioso en el paciente nefrótico es desconocido, aunque la hipótesis es que podría actuar aumentando la acción de los linfocitos $\mathrm{T} 1 \mathrm{y}$ disminuyendo la de los T2. ${ }^{23}$ 
Desde 1980, año de la primera publicación de Tanphaichitr sobre los efectos beneficioso del levamisol en el paciente con recaídas frecuentes o corticodependiente, existe por lo menos una decena de publicaciones en la literatura mundial que obtienen los mismos resultados. ${ }^{24-26}$

La experiencia argentina con esta droga demostró pocos efectos adversos. También aquí estos fueron leves y se trató, en la mayoría de los casos, de rash que remitió al suspender la droga. ${ }^{26}$

Los efectos adversos del levamisol son neutropenia, rash, vasculitis, síntomas gastrointestinales, toxicidad hepática y raramente síntomas neurológicos. En general, son leves. ${ }^{27-30}$

Deberán realizarse al inicio y durante el tratamiento hemograma, hepatograma y función renal para descartar toxicidad.

La ciclofosfamida ha sido usada en pediatría desde 1960; es una droga inmunosupresora con acción directa sobre la médula ósea que causa supresión. Su mecanismo de acción en el síndrome nefrótico aún no es claro.

Luego del tratamiento con ciclofosfamida, un $72 \%$ y un $36 \%$ de los pacientes recaedores frecuentes no presentaron recaídas a los dos y cinco años de seguimiento, respectivamente, mientras que en los pacientes corticodependientes los períodos libres de recaídas a los dos y cinco años son de $40 \%$ y $24 \%$. $^{31,32}$

Hay factores de pronóstico, como la edad y el grado de la corticodependencia, que influyen en la respuesta al tratamiento con ciclofosfamida.

En el estudio de Vester y col., los niños menores de 5,5 años tienen solo un 9\% de chance de estar en remisión a los 5 años de seguimiento posciclofosfamida, y más del $80 \%$ de ellos recaen en el primer año de tratamiento. En este estudio de 106 niños con lesiones mínimas, prácticamente no existió la leucopenia, hecho que avalaría lo mencionado anteriormente. ${ }^{32}$

Los efectos adversos son infecciones, leucopenia, esterilidad, pérdida de la audición, cistitis hemorrágica (se debe a la acroleína, metabolito de la ciclofosfamida que ejerce una acción tóxica sobre el uroepitelio), es de infrecuente presentación cuando la administración de la droga es oral. En algunos casos, también se han descrito convulsiones.

Pero, fundamentalmente, es una droga en potencia oncogénica, dosis-dependiente, razón por la cual la dosis máxima no puede sobrepasar los $180 \mathrm{mg} / \mathrm{kg} /$ vida. $^{31}$

No existen diferencias de resultados a largo plazo entre el uso de la ciclofosfamida oral y endovenosa. El uso endovenoso está indicado en otras patologías (vasculitis, por ejemplo) y raramente se elige esta vía para los pacientes nefróticos. ${ }^{33}$

Se ha descrito intoxicación hídrica secundaria a la administración por vía parenteral. ${ }^{34}$

Los niños tienen un sistema inmune inmaduro, por lo que responden de forma diferente al tratamiento y también la dosis requerida es menor al calcularse por kilo de peso.

\section{Paciente corticorresistente}

Alrededor de un $10 \%$ de los niños con síndrome nefrótico primario son resistentes a los corticoides y requieren la indicación de terapias alternativas. ${ }^{35}$

Antes de clasificar al paciente como corticorresistente, se deben considerar las siguientes situaciones:

- Falta de adherencia al tratamiento.

- Presencia de infecciones que impidan la remisión.

- Falta de absorción de la droga por edema intestinal.

De ser posible, es necesario intentar descartar con el estudio genético la mutación de los genes involucrados en la etiología de síndrome nefrótico hereditario, como la podocina, $\alpha$ actinina, nefrina y el Wt1. ${ }^{14}$

La biopsia renal está indicada, antes de iniciar otro tratamiento. La lesión histológica más común es la esclerosis glomerular focal y segmentaria (EGFS).

El manejo clínico de estos niños es muy difícil dado que presentan, durante la evolución, complicaciones relacionadas con la proteinuria masiva, el estado nefrótico crónico y el tratamiento inmunosupresor:

- Detención del crecimiento

- Desnutrición

- Edema crónico refractario al tratamiento

- Infecciones

- Hiperlipidemia

- Anemia

- Hipertensión arterial

- Insuficiencia renal.

\section{Primera opción}

Ciclofosfamida $2 \mathrm{mg} / \mathrm{kg} / \mathrm{d}$ (no más de 90 días y una dosis máxima acumulativa de $180 \mathrm{mg} /$ $\mathrm{kg}$ ) y prednisona en días alternos $1,5 \mathrm{mg} / \mathrm{kg}$ o $40 \mathrm{mg} / \mathrm{m}^{2}$ con descenso progresivo y lento de prednisona.

Esta indicación se desprende de la opinión de los expertos de los centros nefrológicos presentes en el consenso 2009-2011 en la sede de la Sociedad 
Argentina de Pediatría (SAP), acordada sobre la base de los datos retrospectivos de más de 30 años de seguimiento de pacientes con este tratamiento, cuyo porcentaje de respuesta positiva (remisión) osciló entre un 40 y un $45 \%$.* $^{*}$

Las principales ventajas de esta droga, en la experiencia mencionada, son una respuesta satisfactoria, su bajo costo y la infrecuente aparición de efectos adversos graves, si bien su uso es controvertido a nivel internacional. ${ }^{36,37}$

Se encuentra en elaboración un trabajo del Comité de Nefrología de la SAP prospectivo, cuyos objetivos son "Evaluar la efectividad al tratamiento con ciclofosfamida en pacientes pediátricos con sindrome nefrótico primario corticorresistente" $\mathrm{y}$ "Evaluar posibles efectos adversos de la droga. Y evaluar la respuesta a los corticoides luego del tratamiento con ciclofosfamida".

\section{Ciclofosfamida sensible}

- Ante nueva recaída, reiniciar el tratamiento con prednisona (de acuerdo con el esquema de recaída).

- Ciclofosfamida resistente: no hay consenso. ción:

Opciones para tener en cuenta en esta situa-

\section{Ciclosporina:}

Actualmente, en la mayoría de los centros nefrológicos del mundo, exceptuando nuestro país, es la droga de primera elección en el paciente corticorresistente en lugar de la ciclofosfamida. ${ }^{38-40}$ Además de su efecto inmunosupresor, la ciclosporina tiene una acción hemodinámica (vasoconstrictora de la arteriola aferente), que, al reducir la presión intraglomerular, disminuye la proteinuria. ${ }^{38} \mathrm{Su}$ efecto antiproteinúrico se debería también a la acción no inmunológica directa que ejerce sobre los podocitos al evitar la degradación de la actina. ${ }^{38-41}$

La dosis indicada es $5 \mathrm{mg} / \mathrm{kg} / \mathrm{d}$ o $150 \mathrm{mg} / \mathrm{m}^{2}$ en dos dosis diarias para lograr niveles séricos de 50 a $150 \mathrm{ng} / \mathrm{ml}$ (medido por inmunoensayo). Puede incrementarse la dosis, pero, si bien tiene un mejor efecto en la reducción de la proteinuria, pueden aumentar los efectos adversos. ${ }^{42}$

Datos recientes muestran que también los síndromes nefróticos de etiología genética pueden beneficiarse con su uso. ${ }^{39}$

La toxicidad renal es el efecto adverso más grave e importante de esta droga.

* Hospitales: Garrahan, Posadas, Gutiérrez, Sor María Ludovica de La Plata, Hospital de Niños de Tucumán y Hospital Municipal del Niño de San Justo.
Principalmente, se debe a cambios histológicos tubulointersticiales y vasculares, que se han publicado en un $30-40 \%$ de pacientes pediátricos tratados con ciclosporina por 12 meses o más. Estos cambios van desde la toxicidad aguda y la vacuolización isomérica de las células epiteliales tubulares hasta la fibrosis intersticial renal en un $80 \%$ de ellos cuando el tratamiento se prolonga por 4 años. ${ }^{42-46}$

Los últimos resultados publicados aportan una remisión de la proteinuria en un $80 \%$ en pacientes corticorresistentes con esclerosis focal. ${ }^{46-48}$

Otras recomendaciones para el tratamiento de pacientes que no responden a la ciclofosfamida son ácido micofenólico, tacrolimos y rituximab. En este sentido, solo hay experiencias con muy poco número de pacientes. ${ }^{48}$

\section{Tratamiento del edema}

Indicaciones generales

- Dieta hiposódica.

- Líquidos medidos para lograr un balance negativo de agua.

- En pacientes con anasarca y con signos clínicos de hipovolemia, infundir albúmina 0,5-1g/ $\mathrm{kg}$ EV en 2 horas, asociada a furosemida 1mg/ $\mathrm{kg} /$ dosis en la mitad y al final de la infusión.

- El uso de diuréticos requiriere internación para un cuidadoso control del paciente ya que podrían producir contracción del volumen intravascular, alteraciones del medio interno y fallo renal.

La primera indicación para el tratamiento del síndrome nefrótico es la dieta hiposódica, que debe ser lo más estricta posible, ya que para estos pacientes es lo más importante, debido a la retención de sodio.

Es necesario medir diariamente el balance de ingresos y egresos de líquidos.

"Si bien no existió una indicación unánime respecto a la ingesta líquida adecuada, esta debería ser medida en los pacientes en recaída o corticorresistentes a fin de impedir el aumento exagerado de los edemas. Es importante controlar la restricción para no provocar hipoperfusión renal e inducir necrosis tubular".

\section{Infusión de albúmina}

La hipoalbuminemia es el principal factor de resistencia a la acción de los diuréticos debido a la poca llegada de droga al túbulo.

La infusión de furosemida mas albúmina potencia el efecto diurético debido, posiblemente, a un aumento de la llegada de la furosemida, junto con la albúmina, al asa de Henle e inhibición 
del cotransportador $\mathrm{Na} / \mathrm{K} / 2 \mathrm{C}$, lo que produce natriuresis. ${ }^{49,50}$

La indicación debe ser precisa; es necesario indicarla en pacientes con signos clínicos y de laboratorio de hipovolemia: mala perfusión, dolor abdominal, anasarca, hemoconcentración, uremia elevada de origen prerrenal, hiponatremia y alcalosis. El aumento de la oncosis, seguido de la infusión, es transitorio y puede provocar cambios hemodinámicos en el paciente, como hipertensión y taquicardia durante la infusión. De ser así, es necesario suspender inmediatamente el tratamiento.

\section{Uso de diuréticos}

\section{Tratamiento del edema refractario}

Los niños que no responden a los corticoides ni a ningún otro inmunosupresor presentan edema crónico, llamado refractario, y suelen beneficiarse en situaciones extremas con el uso de diuréticos.

La aparente resistencia en el síndrome nefrótico a la acción diurética de la furosemida puede ser contrarrestada por la acción conjunta con amilorida, bloqueante de los EcNAs presentes en el túbulo distal.

La administración conjunta de furosemida y amilorida en el edema refractario y crónico mejora la excreción urinaria de $\mathrm{Na}{ }^{49-51}$

Esta indicación debe realizarse solo en niños internados, ya que es necesario monitorear los signos de hipovolemia para evitar complicaciones trombóticas y necrosis tubular. Este riesgo se aumenta con el uso de bloqueantes de la angiotensina (enalapril, losartan).

\section{Bloqueo del sistema renina angiotensina ${ }^{52,53}$}

La dosis indicada en pediatría es la siguiente:

- Enalapril 0,2-0,6 mg/kg/día

- Losartan 0,7-2 mg/kg/día

En los pacientes corticorresistentes está indicado el bloqueo dado que la AgII actúa directamente sobre la función y organización glomerular, estimula la apoptosis y produce la disminución de la nefrina, actina y la ZO-1 (proteínas que componen la estructura podocitaria). Aumenta también la síntesis del factor de crecimiento tumoral beta (TGF $\beta$ ) y la expresión de los receptores At1 (receptores de la AgII) a nivel de la superficie podocitaria.

El resultado de su acción es el aumento de la proteinuria, la esclerosis glomerular y progresión a la insuficiencia renal. ${ }^{54}$

Al comienzo se usa una sola droga y luego, si la dosis no es efectiva en el control de la proteinuria, se puede asociar losartan para bloquear completamente el sistema renina angiotensina.

Es importante tener en cuenta que esta asociación aumenta el riesgo de efectos adversos: hiperkalemia, caída del filtrado glomerular y anemia.

No deben indicarse con niveles de creatinina mayor de $3 \mathrm{mg} / \mathrm{dl}$ o en pacientes con contracción de volumen, y se debe evaluar su indicación en aquellos pacientes que reciban otra medicación que produzca hiperkalemia: beta bloqueantes o inhibidores de la aldosterona.

El control de la hipertensión arterial, la anemia, el estado de hipercoagulabilidad, las infecciones y la hiperlipidemia son los parámetros que, al modificarlos y llevarlos a niveles normales, mejoran la calidad de vida de los pacientes crónicos y retrasan la llegada del fallo renal terminal.

\section{Efectos adversos de los corticoides}

La acción tóxica en los distintos órganos se debe a su uso prolongado; puede ocurrir tanto en el paciente corticodependiente como en el corticorresistente.

\section{- Alteración de la mineralización ósea:}

Los corticoides causan aumento de la reabsorción ósea por estimulación directa de la actividad osteoclástica, reducen el número de osteoblastos y su función, por lo tanto, todos los pacientes bajo tratamiento con corticoides deben recibir calcio y vitamina $\mathrm{D}$.

\section{Tratamiento complementario}

- Calcio: 500-1000 mg/d.

- Vitamina D: $1000 \mathrm{UI} / \mathrm{d}$.

- Protector gástrico.

\section{- Retardo del crecimiento}

Hay una relación directa entre la duración del tratamiento con corticoides y el retardo del crecimiento. Posiblemente, se deba en parte a un retraso de la pubertad.

La dosis acumulativa total también tiene un papel importante en la falla del crecimiento: los niños cuyo debut ha sido antes de los tres años se encuentran más comprometidos en su crecimiento, dado que tienen más recaídas y reciben mayor dosis de corticoides.

Sin embargo, los niños recuperan la velocidad del crecimiento si se suspende el tratamiento con corticoides antes de la pubertad..$^{55}$ 
Mejor crecimiento lineal parecería obtenerse con el uso de corticoides en días alternos. ${ }^{56}$

\section{- Obesidad}

Los pacientes que reciben glucocorticoides en exceso desarrollan el síndrome de Cushing (obesidad central e insulinorresistencia) debido a que los glucocorticoides incrementan la liberación de los ácidos grasos de los adipocitos por estimulación de la lipólisis.

\section{- Cataratas}

Las cataratas capsulares posteriores ocurren en un $10 \%$ de los niños con SN tratados con corticoides. ${ }^{57}$

\section{- Necrosis vascular de la cabeza femoral}

La metilprednisolona reduce el flujo vascular en la cabeza femoral, lo cual resulta en un factor de riesgo que favorece la necrosis ósea.

Esta complicación no es común, pero debe considerarse en los diagnósticos diferenciales ante un paciente que refiera coxalgia, dolor en la articulación de la rodilla o alteración de la marcha inexplicada.

\section{- Hipertensión}

No es común la hipertensión en niños con síndrome nefrótico. Sin embargo, los corticoides, al igual que la ciclosporina, pueden elevar la tensión arterial.

Los corticoides actúan elevando la sensibilidad vascular a la acción de AgII endógena y de las catecolaminas. El aumento de la reabsorción de sodio y agua debido a su acción mineralocorticoidea también contribuye a la fisiopatología de la hipertensión.

\section{Pronóstico del síndrome nefrótico}

El pronóstico lo marca la respuesta inicial al tratamiento con corticoides y la histología renal, que son dos líneas paralelas de análisis. Es decir que los pacientes corticosensibles tienen un excelente pronóstico, ya que la mayoría presenta un patrón histológico renal de lesiones mínimas y no recaen más allá de la pubertad. Los pacientes corticorresistentes, por otro lado, presentan frecuentemente esclerosis segmentaria y focal, y más de un $40 \%$ desarrollan fallo renal terminal dentro de los 5 años del inicio de la enfermedad. ${ }^{35}$

\section{CONCLUSIÓN}

- La causa más frecuente de síndrome nefrótico en pediatría es primaria.

- El patrón histológico más frecuente son las lesiones mínimas.

- La corticosensibilidad inicial es $\pm 90 \%$ y las recaídas luego del debut son frecuentes.

- El pronóstico a largo plazo lo define la respuesta inicial al tratamiento con corticoides.

- El paciente corticorresistente representa un desafío de nuevas terapias que permitan reducir al mínimo la toxicidad y prevenir o enlentecer el camino hacia el fallo renal terminal.

- El algoritmo de tratamiento del SN puede resumirse como se muestra en la Figura 1.

\section{Agradecimientos}

A los Dres. Jorge Ferraris y Javier Zalba. 


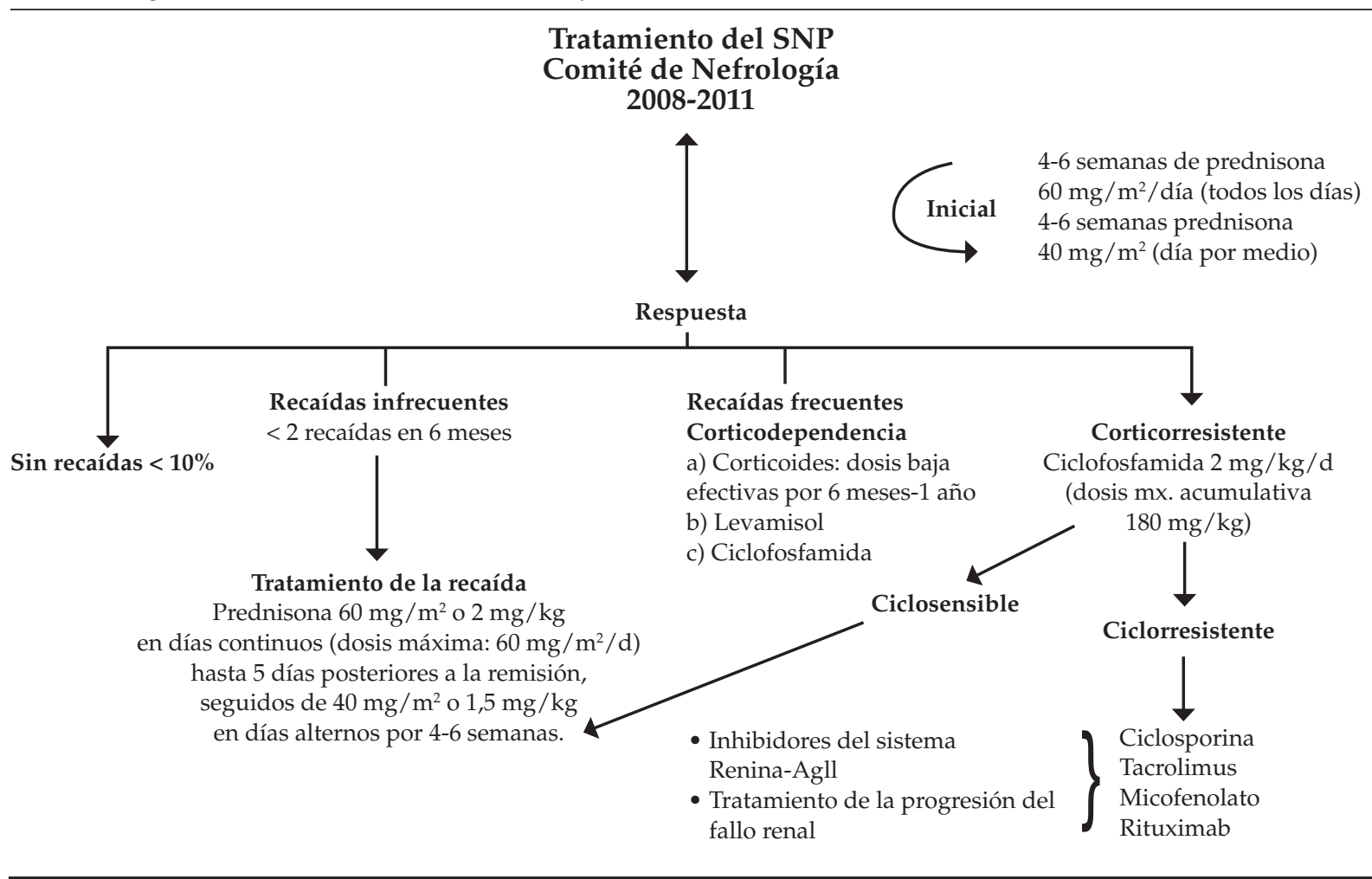

\section{BIBLIOGRAFÍA}

1. Alconcher L, Meneguzzi B. Datos epidemiológicos del síndrome nefrótico en la Argentina. Comité de Nefrología de la Sociedad Argentina de Pediatría. VIII Congreso de la Asociación Latinoamericana de Nefrología Pediátrica. 2008, 23-26 de noviembre, Buenos Aires.

2. Nephrotic Syndrome: Prediction of histopatology from clinical and laboratory characteristics at time the diagnosis. A report of International Study of Kidney Disease in Children. Kidney Int 1978;(13):159-65.

3. Arneil GC, Wilson EH.Cortisone treatment of Nephrosis. Arch Dis Child 1952;27(134):322-8.

4. BarnettHL, Edelmann CM, Greifer I. The primary nephrotic syndrome in children. Identification of patients with minimal change nephrotic syndrome from initial response to prednisone. A report of the international study of kidney disease in children. J Pediatr 1981;98(4):561-4.

5. Hodson EM, Willis NS, Craig JC. Corticosteroid therapy for nephrotic syndrome in children (Review). The Cochrane Library 2010, Issue 4. [Disponible en: www. bibliotecacochrane.com/pdf/CD001533.pdf]. [Consulta: 4 de abril de 2014].

6. Hodson EM, Willis NS, Craig JC. Non-corticosteroid treatment for nephrotic syndrome in children. Cochrane Database Syst Rev 2008;1:CD002290.

7. Gipson DS, Massengill SF, Yao L, Nagaraj S, et al. Management of Childhood Onset Nephrotic Syndrome. Pediatrics 2009;124:747-57.

8. Peña A, Mendizabal S. Síndrome nefrótico en la edad pediátrica. En: Protocolos Diagnóstico Terapéuticos de la Asociación Española de Pediatría. Nefrología Pediátrica, $2^{\text {da }}$ ed. Asociación Española de Pediatría. 2008. [Disponible en: www.aeped.es/sites/default/files/documentos/14_3. pdf]. [Consulta: 4 de abril de 2014].

9. Kidney Disease: improvement global outcome (KDIGO), the 15th Congress of the International Pediatric Nephrology Association (IPNA), held on August 29-September 2, 2010, NY, USA.

10. Stubbe J, Jensen ON, Thiesson HC, Uhrenholt TR, et al. Plasmin in nephrotic urine activates the epithelial sodium channel. J Am Soc Nephrol 2009;20:299-310.

11. Bockenhauer D. Over- or underfill: not all nephrotic states are created equal. Ped Nephrol 2013; 28(8):1153-6.

12. Lagrue G, Xheneumont S, Branellec A, Hirbec G, Weil B. Avascular permeability factor elaborated fromlymphocytes I. Demonstration in patients with nephrotic syndrome. Biomedicine 1975;23:37-40.

13. McCarthy ET, Sharma M, Savin VJ. Circulating factors in Idiopathic Nephrotic Syndrome and focal segmental Glomeruloesclerosis. Clin J Am Soc Nephrol 2010; 5:2115-21.

14. Hinkes BG, Mucha B, Vlangos C, Gbadegesin R, et al. Nephrotic Syndrome in the First Year of Life: Two Thirds of Cases Are Caused by Mutations in 4 Genes (NPHS1, NPHS2, WT1, and LAMB2). Pediatrics 2007;119:e907-19.

15. Koskimies O, Vilska J, Rapola J, Hallman N. Long-term outcome of primary nephrotic syndrome. Arch Dis Child 1982;57:544-8.

16. Kumimasa Y. Subcellular localization of glucocorticoid receptor protein in the human kidney glomerulus. Kidney Int 1999;56:65-73.

17. Schönenberger E, Ehrich JH, Haller H, Schiffer M. The podocyte as a direct target of immunosuppressive agents. Nephrol Dial Transplant 2011;26:18-24.

18. Jayantha UK. Prolonged versus standard steroid therapy for children with a relapsing course of nephrotic syndrome. Pediatr Nephrol 2004;19:C99. 
19. Barnes PJ. Mechanisms and resistance in glucocorticoid control of inflammation. J Steroid Biochem Mol Biol 2010;120:76-85.

20. Tarshish P, Tobin JN, Bernstein J, Edelmann CM Jr. Prognostic significance of the early course of minimal change nephrotic syndrome: report of the International Study of Kidney Disease in Children. J Am Soc Nephrol 1997;8(5):769-76.

21. Laso M, Exeni R, Grimoldi I, Amore A, et al. Primary Nephrotic Syndrome in Children. $12^{\text {th }}$ International Congress of the International Pediatric Nephrology Association. 2001, 1-5 September, Seattle, Washington.

22. Hodson EM, Knight JF, Willis NS, Craig JC. Corticosteroid therapy for nephrotic syndrome in children. Cochrane Database Syst Rev 2004;(2):CD001533.

23. Szeto C, GillespieKM, Mathieeson PW. Levamisole induces interleukin 18 and shifts THI/TH2 balance. Immunology 2000;100:217-22.

24. Tanphaichitr P, Tanphaichitr D, Sureeratanan J. Treatment of nephrotic syndrome with levamisole. J Ped 1980;96:4903.

25. Al-Saran K, Mirza K, Al-Ghanam G, Abdelkarim M. Experience with levamisole in frequently relapsing, steroid-dependent nephrotic syndrome. Pediatr Nephrol 2006;21:201-5.

26. Exeni C, Nava V, Alberto N, Trejomovich V, et al. Eficacia clínica del levamisol en el tratamiento del Síndrome Nefrótico. $4^{\text {to }}$ Congreso Argentino de Nefrología Pediátrica, 2009, 13-16 de mayo, Buenos Aires, Sociedad Argentina de Pediatría; 55 .

27. Rongioletti F, Ghio L, Ginevri F, Bleidl D, et al. Purpura of the ears: a distinctive vasculopathy with circulating autoantibodies complicating long-term treatment with levamisole in children. Br J Dermatol 1999;140:948-51.

28. Palcoux JB, Niaudet P, Goumy P. Side effects of levamisole in children with nephrosis. Pediatr Nephrol 1994;8:263-4.

29. Dubey AK, Gupta RK, Sharma RK. Levamisole induced ataxia. Indian Pediatr 2001;38:417-9.

30. Bulugahapitiya DT. Liver toxicity in a nephrotic patient treated with levamisole. Arch Dis Child 1997;76:289.

31. Latta K, von Schnakenburg C, Ehrich JH. A meta-analysis of cytotoxic treatment for frequently relapsing nephrotic syndrome in children. Pediatr Nephrol 2001;16(3):271-82.

32. Vester U, Kranz B, Zimmermann S, Hoyer PF. Cyclophosphamide in steroid-sensitive nephrotic syndrome: outcome and outlook. Pediatr Nephrol 2003;18(7):661-44

33. Prasad N, Gulati S, Sharma RK, Singh U, Ahmed M. Pulse cyclophosphamide therapy in steroid-dependent nephrotic syndrome. Pediatr Nephrol 2004;19:494-8.

34. Salido M, Macarron P, Hernández-García C, D’Cruz $\mathrm{DP}$, et al. Water intoxication induced by low-dose cyclophosphamide in two patients with systemic lupus erythematosus. Lupus 2003;12(8):636-9.

35. Valentini RP, Smoyer WE. Nephrotic syndrome. En: Kher KK, Schnaper HW, Makker SP, eds. Clinical Pediatric Nephrology. $2^{\text {nd }}$ ed. London: Informa Healthcare; 2007:15593.

36. Plank C,Kalb V,Hinkes B, HildebrandtF, etal. Cyclosporin $\mathrm{A}$ is superior to cyclophosphamide in children with steroid-resistant nephrotic syndrome-a randomized controlled multicentre trial by the Arbeitsgemeinschaft für Pädiatrische Nephrologie. Pediatr Nephrol 2008;23:1483-93.

37. Tarshish P, Tobin JN, Bernstein J, Edelmann CM Jr. Cyclophosphamide does not benefit patients with focal segmental glomerulosclerosis. A report of the International Study of Kidney Disease in Children. Pediatr Nephrol 1996;10(5):590-3.

38. Faul C, Donnelly M, Merscher-Gomez S, Chang YH, et al. The actin cytoskeleton of kidney podocytes is a direct target of the antiproteinuric effect of cyclosporine A. Nat Med 2008;14:931-8.

39. Bensman A, Niaudet P. Non-immunologic mechanisms of calcineurin inhibitors explain its antiproteinuric effects in genetic glomerulopathies. Pediatr Nephrol 2010;25:1197-9.

40. Ingulli E, Singh A, Baqi N, Ahmad H, et al. Aggressive, longterm cyclosporine therapy for steroid-resistant focal segmental glomerulosclerosis. J Am Soc Nephrol 1995;5:1820-5.

41. Plank C, Bensman A, Niaudet P. Non-immunologic mechanisms of calcineurin inhibitors explain its antiproteinuric effects in genetic glomerulopathies. Pediatr Nephrol 2010;25:1197-9.

42. Iijima K, Hamahira K, Tanaka R, Kobayashi A, et al. Risk factors for cyclosporine-induced tubulointerstitial lesions in children with minimal change nephrotic syndrome. Kidney Int 2002;61(5):1801-5.

43. Inoue Y, Iijima K, Nakamura H, Yoshikawa N. Two-year cyclosporin treatment in children with steroid-dependent nephrotic syndrome. Pediatr Nephrol 1999;13(1):33-8.

44. Niaudet P, Habib R, Tete MJ, Hinglais N, Broyer M. Cyclosporin in the treatment of idiopathic nephrotic syndrome in children. Pediatr Nephrol 1987;1(4):566-73.

45. Hamahira K, Iijima K, Tanaka R, Nakamura H, Yoshikawa $\mathrm{N}$. Recovery from cyclosporine-associated arteriolopathy in childhood nephrotic syndrome. Pediatr Nephrol 2001;16(9):723-7.

46. Hamasaki Y, Yoshikawa N, Hattori S, Sasaki S, et al. Cyclosporine and steroid therapy in children with steroidresistant nephrotic syndrome. Pediatr Nephrol 2009;24:217785.

47. Cattran DC, Alexopoulos E, Heering P, Hoyer PF, et al. Cyclosporin in idiopathic glomerular disease associated with thenephrotic syndrome: workshop recommendations. Kidney Int 2007;72:1429-47.

48. Van Husen M, Kemper MJ. New therapies in steroidsensitive and steroid-resistant idiopathic nephrotic syndrome. Pediatr Nephrol 2011;26:881-92.

49. Na KY, Han JS, Kim YS, Ahn C, et al. Does Albumin Preinfusion Potentiate Diuretic Action of Furosemide in Patients with Nephrotic Syndrome? J Korean Med Sci 2001;16:448-54.

50. Doucet A, Favre G, Deschênes. Molecular mechanism of edema formation in nephritic syndrome: therapeutic implications. Pediatr Nephrol 2007;22:1983-90.

51. Rondon-Berrios H. Avances en la fisiopatología del edema en el síndrome nefrótico. Nefrologia 2011;31(2):148-54.

52. Chiurchiu C, Remuzzi G, Ruggenenti P. Angiotensinconverting enzyme inhibition and renal protection in nondiabetic patients: the data of meta-analyses. Am Soc Nephrol 2005;16(supl.1):S58-63.

53. NiaudetP.Steroid-resistantidiopathic nephrotic syndrome in children. En: Avner ED, Harmon WE, Niaudet P, eds. Pediatric Nephrology. Philadelphia: Lippincott Williams \& Wilkins; 2004:557-73.

54. Shankland SJ. The podocyte's response to injury: Role in proteinuria and glomerulosclerosis. Kidney Int 2006;69:213147.

55. Emma F, Sesto A, Rizzoni G. Long-term linear growth of children with severe steroid-responsive nephrotic syndrome. Pediatr Nephrol 2003;18:783-8.

56. Polito C, Oporto MR, Totino SF, La Manna A, Di Toro R. Normal growth of nephrotic children during long-term alternate-day prednisone therapy. Acta Paediatr Scand 1986;75:245-50.

57. Ng JS, Wong W, Law RW, Hui J, et al. Ocular complications of paediatric patients with nephrotic syndrome. Clin Exp Ophthalmol 2001;29(4)239-43. 